\title{
Symmetrical anatomical variant of the anterior belly of the digastric muscle: clinical implications
}

\author{
B. Buffoli ${ }^{1 *}$, D. Lancini ${ }^{1 *}$, M. Ferrari ${ }^{1}$, F. Belotti ${ }^{1}$, P. Nicolai ${ }^{2}$, \\ M. Tschabitscher ${ }^{1}$, R. Rezzani ${ }^{1}$, L.F. Rodella ${ }^{1}$ \\ ${ }^{1}$ Section of Anatomy and Physiopathology, Department of Clinical and Experimental Sciences, \\ University of Brescia, Brescia, Italy \\ ${ }^{2}$ Department of Otorhinolaryngology, Head and Neck Surgery, University of Brescia, Brescia, Italy \\ *Equally share the first authorship
}

[Received: 25 February 2015; Accepted 24 April 2015]

The digastric muscle is an important surgical landmark. Several anatomical variants of the digastric muscle are reported in literature and, in particular, the presence of accessory anterior bellies of the muscle is not uncommon. Here, an unreported symmetrical variant of the digastric muscle was found during a dissection of the suprahyoid region. The dissection showed digastric muscles with an accessory anterior belly, which originated from the anterior belly of muscles in proximity and anteriorly to the intermediate tendon. The accessory bellies were fused together on the midline and were attached with a unique tendon to the inner surface of the mental symphysis. These muscles completely filled the submental triangle. This unreported anatomical variant could be considered an additional contribution to description of the anatomical variants of the digastric muscle, with several implications in head and neck pathology, diagnosis and surgery. (Folia Morphol 2016; 75, 1: 112-116)

Key words: anatomical variant, digastric muscle, suprahyoid region, dissection, gross anatomy

\section{INTRODUCTION}

The digastric muscle is composed of 2 bellies, linked together by an intermediate tendon directed to the great horn of the hyoid bone. The anterior belly runs from the digastric fossa of the mandible to the intermediate tendon, while the posterior belly starts from the intermediate tendon and reaches the mastoid notch of the temporal bone. A branch of the facial artery, the submental artery, vascularises the anterior belly, which is innervated by the mylohyoid branch of the inferior alveolar nerve. The posterior belly of the muscle is vascularised by the occipital artery and the posterior auricular artery and it is innervated by the digastric branch of the facial nerve. Anterior and posterior bellies have different innervation, according to their different embryological origin, from first and second branchial arches respectively. Synergic action of both muscles lowers the mandible, elevates and stabilises the hyoid bone.

Variants of the anterior belly occur in $5.9-53 \%$ of the population $[3,9,13,20]$.

Considering that the digastric muscle is an important surgical landmark and its variations could have significant implications in head and neck surgery, imaging and pathology, we decided to report this case, with the hope it can be useful for clinicians who deal with head and neck dissection and pathology.

\section{MATERIALS AND METHODS}

Head dissection of Caucasian man was performed in the Section of Anatomy. The specimens were fixed in phenol-formalin solution and the arteries were 


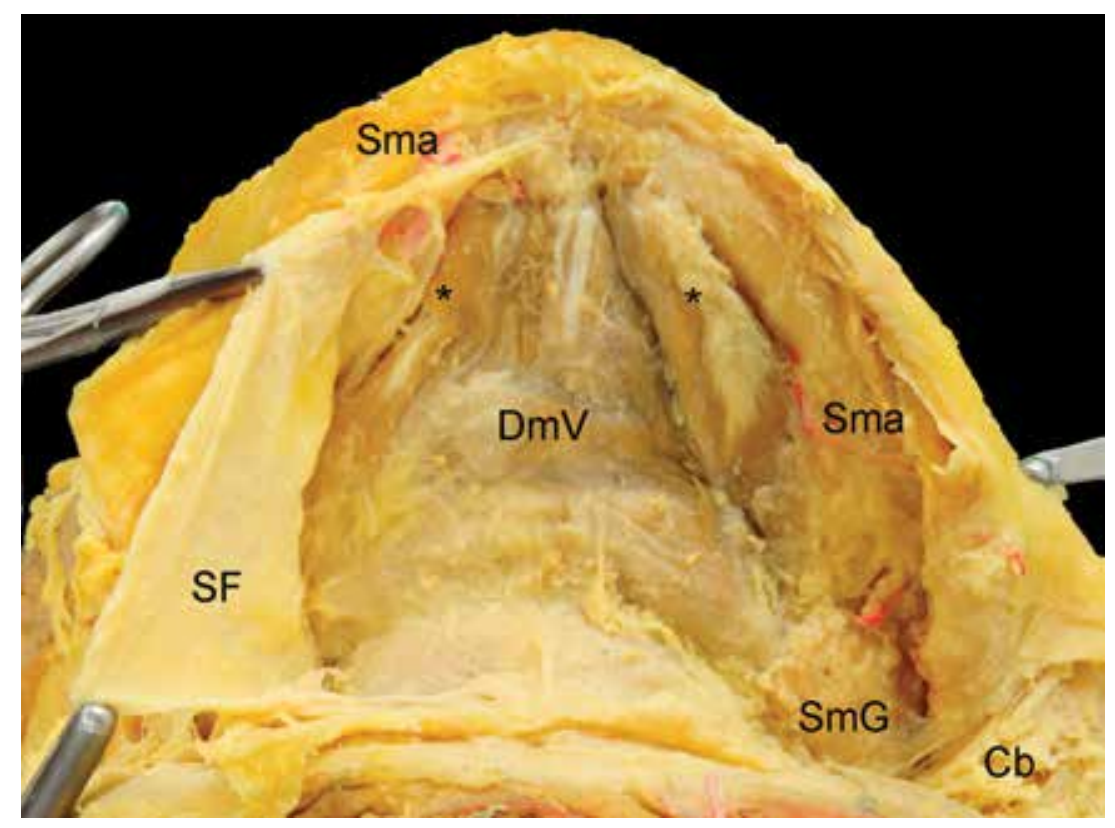

Figure 1. View of the suprahyoid region. The submental triangle is completely filled by muscular tissue, which we have identified as an abnormal digastric muscle; *Anterior bellies of the digastric muscle; $\mathrm{Cb}$ - cervical branch of facial nerve; DmV — digastric muscle variant; SF — superficial fascia; Sma — submental artery; SmG — submandibular gland.

injected with silicone rubber (Dow Corning) red coloured (Mixol).

The dissection was carried out to study the suprahyoid region. First of all, a medial incision of the cutaneous and subcutaneous layers was performed from the mental symphysis to the hyoid bone; platysma muscle was removed and superficial fascia was split and removed showing the digastric muscle. Secondarily, the anterior bellies were reflected (cutting the mandibular insertion) to appreciate the deeper relationship with the mylohyoid muscle.

\section{RESULTS}

A specific anatomic variant was found. Once removed the skin, subcutaneous tissue, platysma muscle and superficial fascia, we observed that the submental triangle was completely occupied by muscles (Fig. 1). Two accessory symmetrical bellies formed this muscle; they originated bilaterally from the anterior bellies, just anteriorly to the intermediate tendon. The accessory bellies were fused along the midline, making a unique muscle which ended with a tendon that reached the mental symphysis in the mandibular inner surface. Once the muscles were reflected, where the accessory bellies joined along the midline, the insertion of the muscles (about $1 \mathrm{~cm}$ ) with the mylohyoid muscle on the mylohyoid raphe was observed (Fig. 2).
Macroscopically, the accessory bellies were comparable in size to the anterior bellies of the digastric muscle, which, in turn, were normal in dimension but slightly lateralised.

\section{DISCUSSION}

Anatomical variants of the digastric muscle are reported in literature $[3,9-12,14,15,19-21]$. These anomalies involve mainly the anterior belly of the muscle and, in particular, the number of the anterior bellies or the presence of accessory bundles originating from a common tendon or from the intermediate tendon $[3,11]$. A quadrification of the anterior belly of the muscle was reported by Celik et al. [3].

Digastric muscle variants have been described more frequently as unilateral $[3,12,14]$ then bilateral $[10,11,21]$ and symmetrical variants of the anterior bellies of the digastric muscle are more rare $[1,11,21]$. Aktekin et al. [1] described 2 accessory anterior bellies, each derived from the homolateral intermediate tendon, crossed together the midline, drawing an " $X$ ", and reached the mandible laterally to the mental symphysis. Similar variant was described by Norton [13] but in this case the posterior halves of the accessory anterior bellies were tendinous and not muscular. Two accessory bellies have been also observed by Liquidate et al. [10], who described 2 bellies arising from the respective digastric fossa 


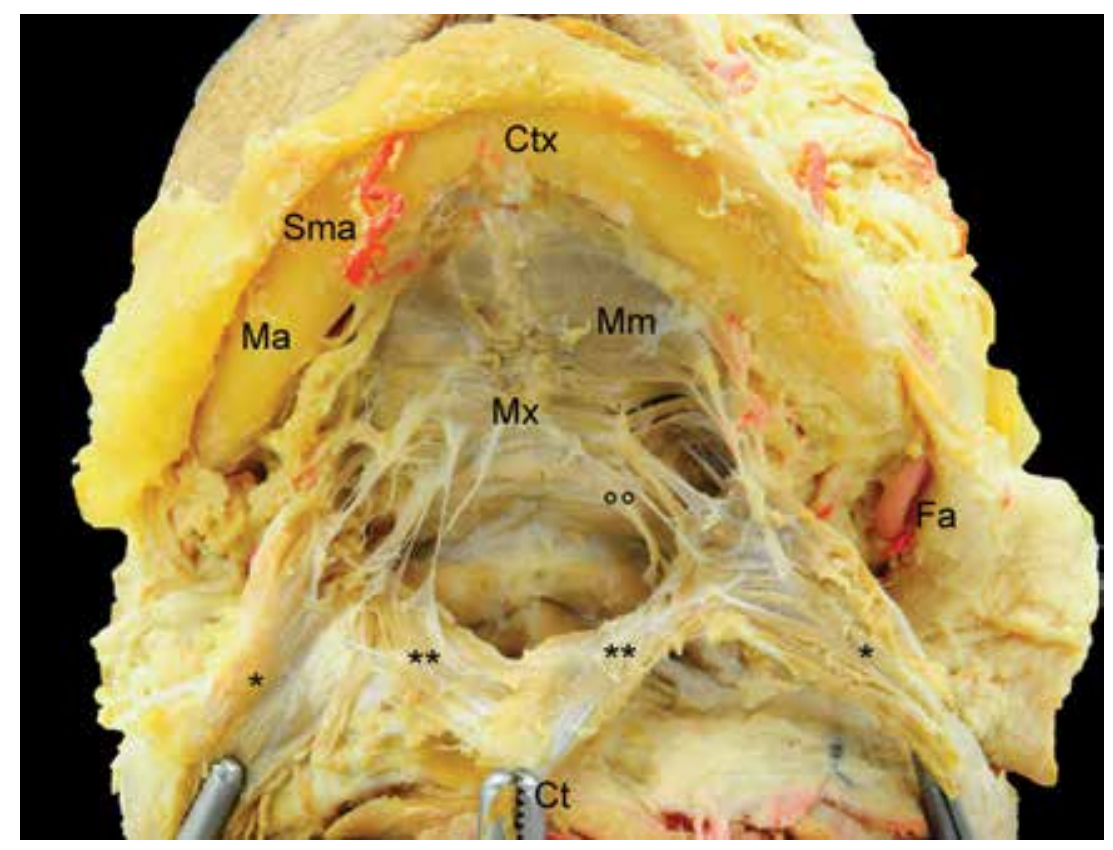

Figure 2. View of suprahyoid region after the dissection of the muscular plane of the digastric muscle (reflected), dividing the muscular bundles. Accessories muscular bellies are visible, originating from the normal bellies of the digastric muscle. These accessory bellies are linked along the midline, forming a common tendon (taken in clamp), that was connected to the mandible. The accessory bellies are connected even to the mylohyoid muscle; *Anterior bellies of digastric muscle; ${ }^{* *}$ Accessory anterior bellies of digastric muscle; ${ }^{\circ}$ Fibrous and muscular connection between digastric muscle and mylohyoid muscle; $\mathrm{Ct}$ - common tendon formed by the accessory anterior bellies; $\mathrm{Ctx}$ — mandibular insertion of the common tendon; $\mathrm{Fa}$ - facial artery; $\mathrm{Ma}$ - mandible; $\mathrm{Mm}$ - mylohyoid muscle; $\mathrm{Mx}$ — insertion of the accessory bellies of digastric muscle on the mylohyoid muscle; Sma — submental artery.

and, fused together, reaching the mylohyoid raphe near the hyoid bone.

We found 2 accessory bellies originated anteriorly to the intermediate tendon of the digastric muscles; they appeared fused along the midline and inserted on the mandibular inner surface (Fig. 3). A similar pattern was seen in other primates such as Tarsius, Saimiri, Macaca, Papio, Gorilla and Pan, in which the main body of the anterior belly of the digastric muscle usually contacts its counterpart (in the midline) for most of its length. Moreover, even if in Cercopithecus the muscle is usually not directly connected to its counterpart, in some specimens was seen an aponeurosis connecting the 2 structures [5].

The anterior bellies of muscles were normal in size but appeared lateral in relation to their anatomical position, probably pushed by the growth of the accessory bellies.

Many authors have proposed a classification for the digastric anterior bellies. De-Ary-Pires et al. [4] classified the digastric muscle by counting the numbers of the anterior bellies, for each side separately, "connected to the mandible or mylohyoid muscle ipsi- and/or contralaterally". They included in this

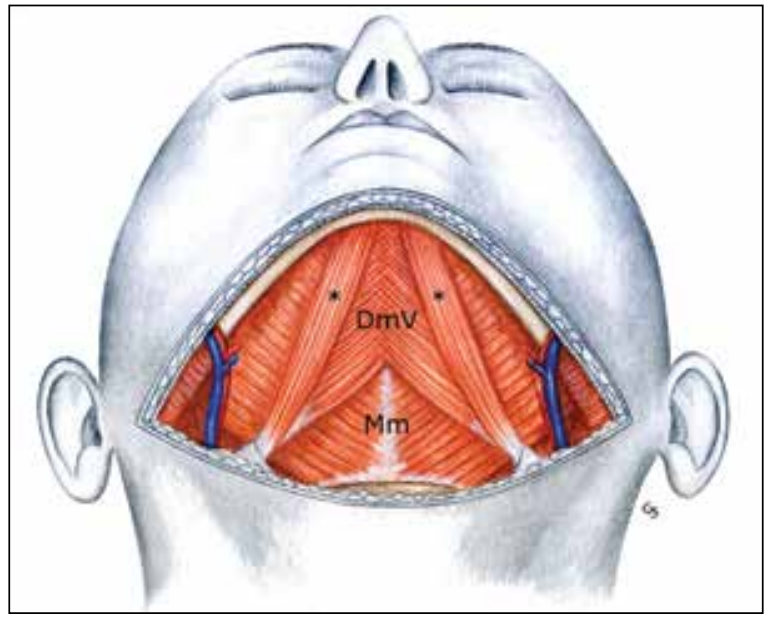

Figure 3. Schematic representation of the symmetrical anatomical variant of the anterior belly of the digastric muscle; ${ }^{*}$ Anterior bellies of the digastric muscle; DmV — digastric muscle variant; $\mathrm{Mm}$ — mylohyoid muscle.

classification even the Macalister's muscle or mentohyoid muscle, as "Anterior Belly Type V". According to this classification, our case could be classed as "Anterior Belly Type II" with the exception that in our finding the accessory belly was connected to the 
contralateral muscle along the midline. Fujimura et al. [6] proposed a way to classify the abnormal anterior belly based on the insertion of the bundles on the mandible or the mylohyoid muscle. According to this criterion, our case could be considered within the classification of the muscles that have their insertion both on the mandible (on the midline in the inner surface of the bone) and on the mylohyoid raphe. Another classification was suggested by Ozgur et al. [14]. They divided the variations of the digastric anterior belly in two groups: Digastric fossa type, in which both origin and insertion (on mandible or hyoid bone) of the accessory bellies are homolateral; and Crossover type, in which the aberrant bellies crossed the midline to reach their insertion, with or without joining the mylohyoid muscle. According to this division, our muscular variant could be considered as a combination of both types, because of the insertion just on the midline.

From an embryological point of view, a possible explanation for this anatomical variant could be a fusion between the first branchial arches. An abnormal maturation of the first branchial arch could also explain the attachment between the digastric muscle and the mylohyoid muscle that have the same origin $[4,16]$. This could also clarify the function of the accessory digastric bellies in supporting the mylohyoid muscle during its physiological functions, such as the mechanic processes of deglutition and mastication. Moreover, considering that the dimension of the accessory bellies was similar to the anterior bellies of the digastric muscle, we can suppose that this anatomical variant could have a real functional implication in the physiological processes.

Anomalies in the vascularisation and innervation of the digastric muscle are also described in literature. Kawai et al. [8] reported an additional innervation from the stylohyoid branch of the facial nerve. This variant was not observed in our case, in which we found usual nerve and artery: the mylohyoid nerve and the submental artery.

From a clinical point of view, normal digastric muscle represents an important landmark for head and neck surgeons; therefore, a variant of the muscle has to be recognised by the surgeons who deal with this region. The main implication that clearly appears from our study was that the accessory muscles completely filled the submental triangle (the space delimited by the hyoid bone posteriorly, the anterior bellies laterally and the mandible anteriorly).
This space contains the Level IA lymph nodes (following Robbins' classification); these lymph nodes can harbour metastases from cancers arising from the floor of mouth, anterior oral tongue, anterior mandibular alveolar ridge, and lower lip [17]. Secondly, the anterior bellies mark the medial limit of the submandibular triangle (limited in other directions by the stylohyoid muscle posteriorly and superiorly by the mandible), also known as Level IB. The presence of an accessory digastric belly could modify the topographic anatomy in a neck dissection including Level I.

Other surgical implications of this variant can be found in plastic and reconstructive surgery. Atamaz Pinar et al. [2] wrote about the use of a sub-mental flap to reconstruct intra-oral or facial defects. This flap is vascularised by the submental artery and contains the anterior belly of the digastric muscle. The digastric muscle can also be used to restore the depressor function of the lower lip resulting from a palsy of the marginal mandibular branch of the facial nerve. According to Tan [18], who described a surgical procedure called ABDMT (Anterior Belly of Digastric Muscle Surgical Transfer) - the intermediate tendon of the digastric muscle could be sutured to the lateral angle of the lower lip to restore the symmetry lost after the palsy. An abnormal muscle in this region could make more challenging to harvest the flap and transfer the muscular bundle.

Anatomical variant of the digastric muscle should be also well-known to head and neck radiologists. Larsson and Lufkin [9] and, more recently, Guelfguat et al. [7] underlined that an abnormal digastric muscle could be easily mistaken for a pseudomass or a pathological lymph node. Other implications can be found in the diagnostic approach to lymphadenopathies of the submental and submandibular triangles. For example, in presence of a similar muscular variant, a lymph-node biopsy for the diagnosis of lymphoma can be obstructed, delaying the diagnostic process.

\section{CONCLUSIONS}

In conclusion, our work describes an unreported bilateral and symmetrical variant of the anterior bellies of the digastric muscles and it could be considered an additional contribution to the description of the anatomical variants of this muscle, with several implications in head and neck pathology, diagnosis and surgery. 


\section{ACKNOWLEDGEMENTS}

The authors thank the donors of the cadavers for their indispensable contribution to this anatomical study.

\section{REFERENCES}

1. Aktekin M, Kurtoğlu Z, Oztürk AH (2003) A bilateral and symmetrical variation of the anterior belly of the digastric muscle. Acta Med Okayama, 57: 205-207.

2. Atamaz Pinar Y, Govsa F, Bilge $O$ (2005) The anatomical features and surgical usage of the submental artery. Surg Radiol Anat, 27: 201-205. doi: 10.1007/s00276005-0317-8.

3. Celik HH, Aldur MM, Ozdemir B, Akşit MD (2002) Abnormal digastric muscle with unilateral quadrification of the anterior belly. Clin Anat, 15: 32-34. doi: 10.1002/ca.1088.

4. De-Ary-Pires B, Ary-Pires R, Pires-Neto MA (2003) The human digastric muscle: patterns and variations with clinical and surgical correlations. Ann Anat, 185: 471-479. doi: 10.1016/S0940-9602(03)80110-3.

5. Diogo R, Wood B (2011) Soft-tissue anatomy of the primates: phylogenetic analyses based on the muscles of the head, neck, pectoral region and upper limb, with notes on the evolution of these muscles. J Anat, 219: 273-359. doi: 10.1111/j.1469-7580.2011.01403.x.

6. Fujimura A, Onodera M, Feng XY, Osawa T, Nara E, Nagato $S$, Matsumoto Y, Sasaki N, Nozaka Y (2003) Abnormal anterior belly of the digastric muscle: a proposal for the classification of abnormalities. Anat Sci Int, 78: 185-188.

7. Guelfguat M, Nurbhai N, Solounias N (2001) Median accessory digastric muscle: radiological and surgical correlation. Clin Anat, 14: 42-46. doi: 10.1002/ /1098-2353(200101)14:1<42::AID-CA1007>3.0.CO;2-O.

8. Kawai K, Koizumi M, Honma S, Tokiyoshi A, Kodama K (2003) Derivation of the anterior belly of the digastric muscle receiving twigs from the mylohyoid and facial nerves. Ann Anat, 185: 85-90. doi: 10.1016/S09409602(03)80018-3.
9. Larsson SG, Lufkin RB (1987) Anomalies of digastric muscles: CT and MR demonstration. J Comp Assist Tomogr, 11: 422-425.

10. Liquidato BM, Barros MD, Alves AL, Pereira CSB (2007) Anatomical study of the digastric muscle: variations in the anterior belly. Int J Morphol, 25: 797-800.

11. Mangalagiri AS, Razvi MR (2009) Variations in the anterior belly of digastric. Int J Health Sci (Qassim), 3: 257-262.

12. Michna $H$ (1989) Anatomical anomaly of human digastric muscles. Acta Anat (Basel), 134: 263-264.

13. Norton MR (1991) Bilateral accessory digastric muscles. $\mathrm{Br}$ J Oral Maxillofac Surg, 29: 167-168. doi: 10.1016/02664356(91)90030-9.

14. Ozgur Z, Govsa F, Ozgur T (2007) The cause of the difference in the submental region: aberrant muscle bundles of the anterior belly of the digastric muscle. J Craniofac Surg, 18: 875-881. doi: 10.1097/scs.0b013e31806844da.

15. Peker T, Turgut HB, Anil A (2000) Bilateral anomaly of anterior bellies of digastric muscles. Surg Radiol Anat, 22: 119-121.

16. Radlanski RJ, Renz H, Tabatabai A (2001) Prenatal development of the muscles in the floor of the mouth in human embryos and fetuses from 6.9 to $76 \mathrm{~mm}$ CRL. Ann Anat, 183: 511-518.

17. Robbins KT, Clayman G, Levine PA, Medina J, Sessions R, Shaha A, Som P, Wolf GT (2002) Neck dissection classification update: revisions proposed by the American Head and Neck Society and the American Academy of Otolaryngology-Head and Neck Surgery. Arch Otolaryngol Head Neck Surg, 128: 751-758.

18. Tan ST (2002) Anterior belly of digastric muscle transfer: a useful technique in head and neck surgery. Head Neck, 24: 947-954. doi: 10.1002/hed.10150.

19. Turan-Ozdemir S, Oygucu IH, Kafa IM (2004) Bilateral abnormal anterior bellies of digastric muscles. Anat Sci Int, 79: 95-97.

20. Uzun A, Aluclu A, Kavakli A (2001) Bilateral accessory anterior bellies of the digastric muscle and review of the literature. Auris Nasus Larynx, 28: 181-183. doi: 10.1016/ S0385-8146(00)00100-0.

21. Ziółkowski M, Marek J, Kłak A (1984) The human digastric muscle in the fetal period. Folia Morphol, 43: 243-249. 